\title{
From sound to meaning: Phonology-to-Semantics mapping in visual word recognition
}

\author{
Simona Amenta ${ }^{1} \cdot$ Marco Marelli $^{2} \cdot$ Simone Sulpizio ${ }^{3,4}$
}

Published online: 29 August 2016

(C) Psychonomic Society, Inc. 2016

\begin{abstract}
In the present study, the role of phonological information in visual word recognition is investigated by adopting a large-scale data-driven approach that exploits a new consistency measure based on distributional semantics methods. A recent study by Marelli, Amenta, and Crepaldi (2015) showed that the consistency between an orthographic string and the meanings to which it is associated in a large corpus is a relevant predictor in lexical decision experiments. Exploiting irregular mappings between orthography and phonology in English, we were able to compute a phonology-to-semantics consistency measure that dissociates from its orthographic counterpart and tested both measures on lexical decision data taken from the British Lexicon Project (Keuleers et al., 2012). Results showed that both orthography and phonology are activated during visual word recognition. However, their contribution is crucially determined by the extent to which they are informative of the word semantics, and phonology plays a crucial role in accessing word meaning.
\end{abstract}

Keywords Phonology-semantics consistency ·

Orthography-semantics consistency $\cdot$ Visual world recognition $\cdot$ Distributional semantic models

Simona Amenta

simona.amenta@gmail.com

1 Center for Mind/Brain Sciences, University of Trento, Corso Bettini 31, 38068 Rovereto, TN, Italy

2 Department of Experimental Psychology, Ghent University, Ghent, Belgium

3 Department of Psychology and Cognitive Science, University of Trento, Rovereto, Italy

4 Fondazione Marica De Vincenzi ONLUS, Rovereto, Italy

\section{Introduction}

While reading, people extract orthographic information from print and map it to phonological and semantic information. But can word meaning be accessed through phonology?

The investigation of the above question goes back to the last century: Empirical research on expert readers has collected multiple contrasting evidence suggesting either that word meaning is accessed by means of orthographic information with no reference to phonology or that phonological information may drive access to semantics apart from and beyond orthographic information. In this paper, we offer fresh empirical evidence for the latter view by adopting a data-driven, atheoretical approach.

Going back at the origins of the debate, we immediately find two contrasting positions: 1) the direct access hypothesis, which proposed that word meaning is accessed directly from word's orthography without reference to its phonology (Seidenberg, 1985); and 2) the phonological mediation hypothesis (Van Orden, 1987), which assumed that word meaning is accessed proceeding from form to sound. The two hypotheses were taken into consideration as two separate, although interacting, routes in the Dual Access Theory (Seidenberg, 1995), where both phonological mediation and orthographic-direct access are possible routes to word meaning (see also Harm \& Seidenberg, 2004 for a discussion of the joint determination of word meaning by phonological and visual processes).

The relationship between orthography and phonology was tested mainly by relying on two strategies (Lesch \& Pollastek, 1993): the manipulation of spelling/sound regularities, and the manipulation of visual and phonological similarity. The first approach is based on the assumption that if word recognition proceeds from spelling to sound to meaning, then spelling-tosound regularity should affect word identification. Regular 
words, i.e., words that preserve the most common orthography-to-phonology mapping, such as "save," should be processed faster than irregular words, such as "have." This line of research however produced mixed results: Some studies reported a regularity effect on all words (Van Orden, 1987), whereas others only for low-frequency words (Jared \& Seidenberg, 1991).

Stronger evidence of phonological mediation came from the second approach (Coltheart, Avons, Masterson, \& Laxon, 1991; Van Orden, 1987; Van Orden, Johnston, \& Hale, 1988), which involved the study of homophones with semantic tasks (categorization judgment and semantic decision). The core ideas of these studies is that, if the homophone of a word is incorrectly accepted as member of the target word category, then this means that phonology is involved in word recognition and comprehension (homophony effect). A large homophone effect was observed for words (Van Orden, 1987; Braun, Hutzler, Münte, Rotte, Dambacher, Richlan \& Jacobs 2015) and nonwords (Van Orden et al., 1988; Braun et al., 2015), suggesting that not only phonological coding is rapid but also automatically activated (along with orthographic information) and used for accessing word meaning. Although replicated with other tasks (e.g., masked priming in silent reading, Perfetti, Bell, \& Delaney, 1988), the homophone effect was found to be significantly sensitive to list composition (Brysbaert, Praet, \& d'Ydewalle, 1990) and lexical frequency (Jared \& Seidenberg, 1991).

All paradigms used until now have showed to be sensitive to a large number of intervening factors (see Frost, 1998, for a complete discussion on this issue), leaving open the possibility that the phonological effects reviewed above are a byproduct of methodological choices. Thus, it is necessary to test this problem on a larger number of items where frequency is not varied factorially. Therefore, we need a new way to look at the core of the problem, relying on direct measurement of the orthography-semantics vs. phonology-semantics mapping, and with an experimental design that takes into consideration possible relevant predictors on large-scale data.

Our approach moves from a recent work by Marelli, Amenta, and Crepaldi (2015), who have proposed a new measure to address the role of orthography in accessing word meaning. Orthography-Semantics Consistency (OSC) is a corpus-based measure that quantifies the relationship between a letter string and the meanings of all the words in a corpus that share that sequence. To compute this measure the authors exploited methods from distributional semantics (Turney \& Pantel, 2010), that can provide cognitively-sound estimates of semantic association (e.g., LSA, Landauer \& Dumais, 1997; HAL, Lund \& Burgess, 1996). This approach is based on the assumption that the meaning of a word can be approximated by the way that it co-occurs with other words in the lexicon. In a Distributional Semantic Model (DSM), word meanings are represented as vectors that are derived from these co-occurrences. The more two words tend to occur with the same set of other words (i.e., in similar contexts), the more their vectors will be close, the more their meanings will be considered to be similar. Geometrically, this amounts to measuring the cosine of the angle formed by the two vectors: The more similar the vectors, the smaller the angle between them, the higher their cosine. Mathematically, OSC is computed as the frequency-weighted average semantic similarity between the vector of a word and the vectors of all words that contain that very same word (see below for a detailed description). Marelli and colleagues (2015) showed that OSC scores could explain reaction times in the lexical decision task on 1,821 words randomly taken from the British Lexicon Project (BLP) (Keuleers, Lacey, Rastle \& Brysbaert, 2012), over and above family size, word length and frequency effects. This measure allows to look directly into the link between orthography and meaning and has two main merits: 1) it is quantitatively and automatically determined, and gives back quantitative and easy to interpret information; and 2) it is mainly a-theoretical, because it is based on observed quantitative relationships between words in a given text corpus. Note that OSC is computed in a semantic space, thus, it is intrinsically a semantic measure that encompasses both the relationship between orthographic units and distributed representations of word meanings. These characteristics make OSC a powerful tool to extend previous investigations to the phonology-to-semantics relation and test whether phonological information plays any role in activating word meaning.

In fact, given the availability of phonological transcriptions in corpora, a Phonology-Semantics Consistency measure (PSC) can be computed. Moreover, exploiting irregularities between orthographic and phonological mapping (e.g., the sublexical string -ough is pronounced differently in different lexical contexts, such as "rough," "dough," "through," "thought," etc.), it is possible to differentiate between OSC and PSC and use these measures as predictors of, respectively, orthography-to-semantics and phonology-to-semantics relation and their interplay in visual word recognition.

In this study, we will compute PSC and then we will test it, along with OSC, in a pool of words extracted from the BLP. As it is, the BLP offers lexical decision latencies to unprimed words, thus we will be able to test our measures in a simple task with no experimental manipulations (no masks, no primes, no SOA-related effects, etc.). Moreover, with a multiple regression design we will be able to control for item properties that were deemed to affect the influence of phonological information (e.g. frequency, distribution, etc.). With this procedure, we will be able to track the dynamics of orthographic and phonological information in word recognition. We will show that both phonological and orthographic information are used to access semantics; however, the effect of the 
orthography-to-semantics mapping is largely mediated by phonological aspects, speaking for the crucial role of phonology in accessing word meaning.

\section{Methods}

We considered a set of 533 English words containing grapheme sequences that are associated to multiple phonological forms in language usage. For example, the initial string leais pronounced differently in leather (leð ), learn (13n), and leaf (li f). This criterion was aimed at maximizing the difference between the semantic information associated to the orthographic form vis-a-vis the phonological form, as captured by the measures of orthography-semantics consistency (OSC) and phonology-semantics consistency (PSC). Experimental items were also included in the BLP (Keuleers et al., 2012), from which we extracted trial-level response times (RTs) in lexical decision. Table 1 reports descriptive data for the experimental set. Frequency values were extracted from SUBTLEX-UK (van Heuven, Mandera, Keuleers, \& Brysbaert, 2014), whereas the English Lexicon Project (ELP, Balota, Yap, Hutchison, Cortese, Kessler, Loftis \& Treiman, 2007) was the source of average bigram frequency, number of phonemes, number of syllables, orthographic neighborhood size, and phonological neighborhood size. As additional variables, we considered the measure of semantic density proposed by Marelli and Baroni (2015) and a measure of orthography-phonology consistency, defined as the average phonological Levenshtein-distance between a target word and its orthographic relatives. The former was based on the estimates from the DSM described below, whereas the latter was obtained by using CELEX phonological annotations (Baayen, Piepenbrock, \& Gulikers, 1995).

To compute PSC and OSC we followed the same procedure and sources described in Marelli et al. (2015). OSC is defined as:

$\operatorname{OSC}(t)=\frac{\sum_{x=1}^{k} \cos \left(\vec{t}, \overrightarrow{r_{x}}\right) * f_{r_{x}}}{\sum_{x=1}^{k} f_{r_{x}}}$

Where $t$ is the target word, $r_{x}$ each of its $k$ orthographic relatives, and $f_{r x}$ the corresponding frequencies. Note that the equation can be rewritten in probabilistic terms:

$\operatorname{OSC}(t)=\sum_{x=1}^{k} p_{x}{ }^{*} \cos \left(\vec{t}, \overrightarrow{r_{x}}\right)$

Where $p_{x}$ is the probability of a given relative in the considered relative set. Expressed in these terms, OSC represents the expected semantic similarity between a word and its orthographic relatives.
We considered, as orthographic relatives, each word containing the target (e.g., "flux" for "flu") from a list, including the top 30,000 most frequent content words (adjectives, nouns, verbs, adverbs) in a 2.8-billion-words corpus (a concatenation of UkWaC, Wikipedia, and BNC). The same corpus was employed to compute $\cos (t, s)$, that is, the semantic similarity between a target and each of its relatives, defined in geometrical terms through distributional semantics techniques. In the present study, we built a distributional model on the basis of the above described corpus (part-of-speech tagged and lemmatized). We focused on word-to-word cooccurrences involving the top $30 \mathrm{k}$ most frequent content words, collected using a 5-word window; raw counts were reweighted using Positive Pointwise Mutual Information (Church \& Hanks, 1990), and we reduced matrix dimensions by means of Non-negative Matrix Factorization (Arora, Ge, \& Moitra, 2012), setting the number of dimensions of the reduced space to 350 . These parameters were adopted because they were shown to produce high quality semantic spaces in previous studies (Bullinaria \& Levy, 2007). For model implementation we relied on the freely available DISSECT toolkit (Dinu, The Pham, \& Baroni, 2013).

We followed the same procedure to obtain PSC, which was formalized exactly as OSC. The only difference in the computation lays in the definition of the relatives that, in the case of PSC, were phonologically defined: A phonological relative is a word that, in its phonological form, contains the phonological sequence of the target word (e.g., "cognac"-/ k njæk/ for “yak"-/'jæk/). Phonological annotations were obtained from CELEX (Baayen et al., 1995).

The interaction between OSC and PSC was tested in a mixed-effects model (Baayen, Davidson, \& Bates, 2008) with lexical-decision RTs as dependent variable, ${ }^{1}$ and random intercepts for items and participants. All the measures listed in Table 1 were considered as potential predictors. Previous RTs and item order were included as control variables. Both RTs and frequency values were log-transformed. We started the analysis from a model, including all predictors, that were subsequently removed when their inclusion did not significantly contributed to model fit (as evaluated by goodness-of-fit Chisquare tests). The reliability of the obtained results was tested through model criticism (Baayen, 2008), i.e., outlying datapoints were removed on the basis of the model residuals (adopting $2 \mathrm{SD}$ as a threshold). The reported results are those of the model refitted after outlier removal.

\footnotetext{
${ }^{1}$ We tested PSC and OSC using the BLP for consistency with our source corpora and annotation (mostly focusing on British English). However, consistent results were observed when analyzing data from the ELP, showing that the same effect can be observed on American English.
} 
Table 1 Summary of the distribution of the predictor variables in the considered item set

\begin{tabular}{|c|c|c|c|c|c|c|}
\hline Predictor & Min & 1st quartile & Median & 3rd quartile & Max & Mean (SD) \\
\hline Word frequency & 7 & 614 & 2196 & 8931 & 1162474 & $14540(61252)$ \\
\hline Average bigram frequency & 100 & 1045 & 1544 & 2135 & 5836 & $1632(786)$ \\
\hline Number of phonemes & 2 & 3 & 4 & 5 & 8 & $3.97(1.11)$ \\
\hline Number of syllables & 1 & 1 & 1 & 2 & 2 & $1.37(0.48)$ \\
\hline Number of characters & 2 & 4 & 5 & 6 & 8 & $4.96(1.32)$ \\
\hline Orthographic neighborhood size & 0 & 1 & 5 & 10 & 24 & $6.24(5.66)$ \\
\hline Phonological neighborhood size & 0 & 3 & 11 & 21 & 45 & $13.42(11.37)$ \\
\hline Orthography-phonology consistency & 0 & 1.83 & 3.24 & 4.48 & 8.01 & $3.17(1.79)$ \\
\hline Semantic density & .46 & .59 & .66 & .74 & .94 & $.67(.09)$ \\
\hline OSC & .02 & .42 & .74 & .89 & .99 & $.65(.31)$ \\
\hline PSC & .01 & .28 & .68 & .87 & .99 & $.59(.32)$ \\
\hline
\end{tabular}

Lexical frequencies are based on SUBTLEX-UK (201,335,638 tokens)

\section{Results}

Table 2 reports the results of the finally obtained model. Along with the interaction between PSC and OSC, we observed negative effects of frequency and item order (larger values correspond to shorter RTs) and a positive effect of number of syllables, phonological neighborhood size, previous RTs, and semantic density (larger values correspond to longer RTs). The PSC-by-OSC interaction is represented in Fig. 1, which shows that the two effects contribute to word recognition, with higher value on either measures corresponding to shorter RTs. However the larger impact of a given predictor is observed when the other has low values: OSC has a stronger effect for low PSC values, and vice versa. ${ }^{2}$

To better understand the interplay between the two variables, we further performed a mediation analysis (Tingley, Yamamoto, Hirose, Keele, \& Imai, 2014). We tested the models presented in Fig. 2. As shown by Table 3, results indicate a mediation of PSC on the direct effect of OSC (Fig. 2a), whereas the alternative hypothesis (OSC mediating PSC; Fig. 2b) is not supported. Sensitivity analysis indicates that the estimated mediation effect equals zero when $\rho$ (the correlation between the error terms of the mediation and outcome models) equals -0.2 (Tingley et al., 2014).

\section{Discussion}

We informed the old issue of the role of phonology in visual word recognition using a novel approach. To do so, we borrowed a measure that was recently proposed for capturing the relationship between orthographic form and meaning

\footnotetext{
${ }^{2}$ Notwithstanding our efforts in composing the item list, a correlation between PSC and OSC was still found in our item set $(r=.69)$. However, the results are confirmed when applying decorrelation techniques to our measures, speaking for the reliability of the reported results.
}

(OSC), and we adapted it to encompass phonological information (PSC). Both OSC and PSC were tested as predictors of lexical decision latencies on a pool of words extracted from the BLP.

The results we found indicate that the two sources of information are active during the task: Both the mapping between phonology and semantics and the mapping between orthography and semantics contribute to word recognition. However, the interaction we found indicates a more complex interplay between the two variables, and the mediation analysis suggests that a large part of the effect of OSC is mediated by PSC: phonology plays a crucial role in accessing word meaning. This is a direct evidence that readers use phonological information to activate semantics, even when the task would not apparently require it (visual word recognition). It is worth noting that our data were obtained analyzing subjects' responses to a simple task (unprimed lexical decision), where the processing and/or disambiguation of homophones or

Table 2 Summary of the results of the mixed-effects analysis

\begin{tabular}{lrlrl}
\hline Predictor & Estimate & Std. error & t-value & $p$ value \\
\hline Intercept & 5.5611 & 0.0431 & 128.44 & 0.0001 \\
Item order & -0.0001 & 0.0001 & -12.64 & 0.0001 \\
Previous RT & 0.1524 & 0.0051 & 30.64 & 0.0001 \\
Word frequency & -0.0305 & 0.0014 & -21.62 & 0.0001 \\
Phonological neighborhood size & 0.0005 & 0.0003 & 2.18 & 0.0294 \\
Number of syllables & 0.0292 & 0.0071 & 4.18 & 0.0001 \\
Semantic density & 0.0619 & 0.0256 & 2.34 & 0.0197 \\
OSC & -0.0401 & 0.0166 & -2.71 & 0.0069 \\
PSC & -0.0704 & 0.0231 & -2.84 & 0.0047 \\
OSC by PSC & 0.0645 & 0.0294 & 2.28 & 0.0232 \\
\hline
\end{tabular}

The lmerTest package (Kuznetsova, Brockhoff \& Christensen, 2015) was used to compute $p$ values. Predictors that are not reported in the table had been removed from the analysis, because they did not significantly contribute to the model fit 


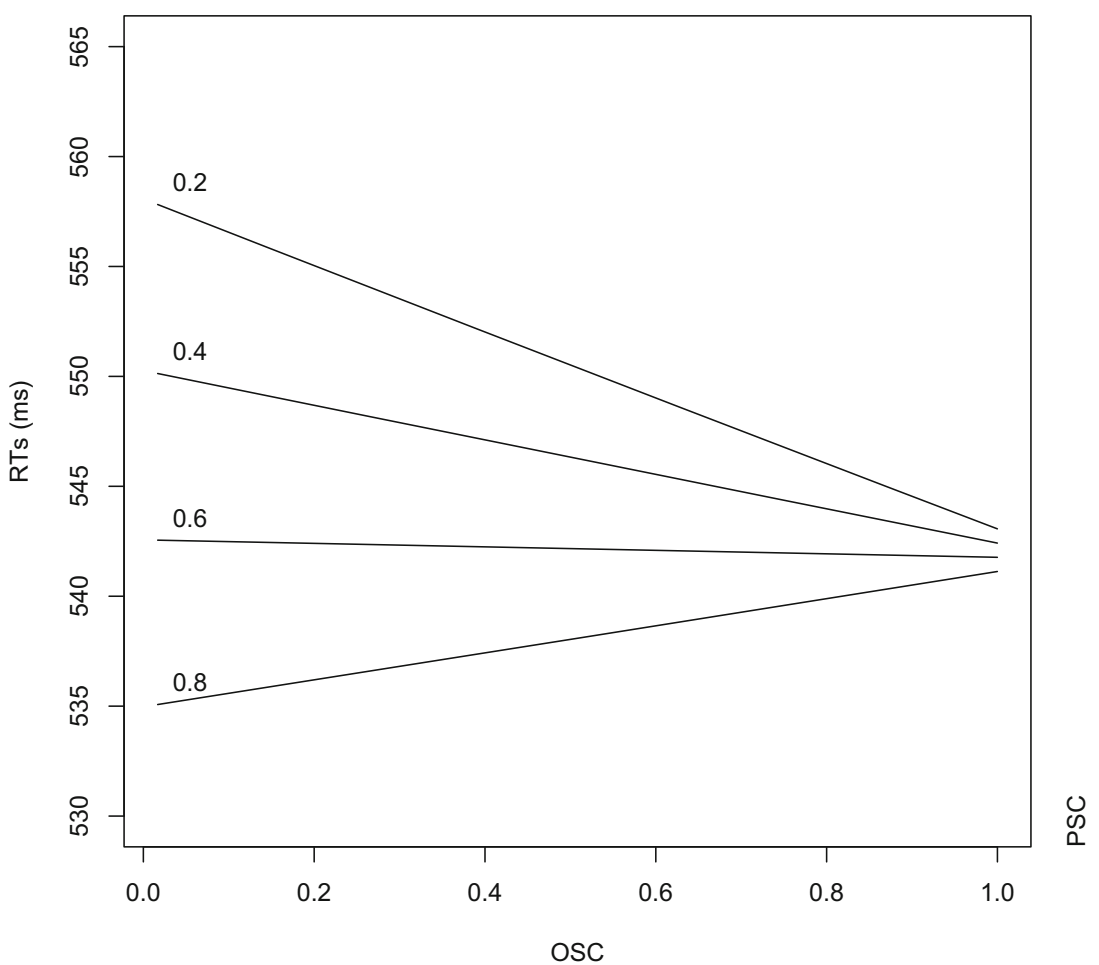

Fig. 1 Interaction between OSC and PSC as predicted by the mixed-effects model. The change of the OSC effect at different levels of PSC is represented by the different regression lines

pseudo-homophones primes was not involved. As a result, we were able to observe participants' responses to lexical stimuli without them being influenced or biased by strong experimental manipulations (that may have led to, e.g., the explicit activation of phonological information through the presentation of homophones). In other words, in this study we observe the influence of phonology not as an effect elicited by some specific experimental manipulation but as an inherent component of the reading process.

One may argue that our experiment is biased by the inclusion of spelling-to-sound inconsistencies, because we selected our item words to maximize the distance between phonological and orthographic information. However, PSC (and OSC as well) remain a simple measure of certain word properties extracted from corpora. Moreover, we analyzed a limited sample of words handpicked from the BLP, a large resource that
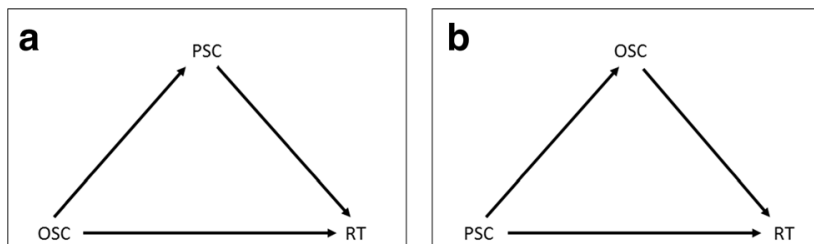

Fig. 2 Model tested in the mediation analysis. Panel 2a represents the hypothesis that the effect of OSC on the Reaction Times (RTs) is mediated by PSC. Panel $2 b$ shows the hypothesis that the effect of PSC on RTs is mediated by OSC contains subjects' responses to a massive number of items. This ensures that our data are free from any possible artifact due to explicit strategies originated by list composition, task requirements, or direct involvement of phonological manipulations. Participants in the BLP study evaluated our experimental stimuli among a huge number of other different words.

The reported results are also informative from a modelling perspective. As noted in the Introduction, both OSC and PSC are intrinsically semantic measures that put in relation the meanings of words that share either (part of) their orthographic or phonological form. Therefore, the interaction between PSC and OSC indicates that word meaning could be accessed following two paths (orthography-to-meaning vs. phonology-to-meaning): We observed that when the mapping between phonological form and meaning (PSC) is less consistent, then OSC helps more in the identification of the word. Our results could be interpreted in spirit with those models of language processing that propose that two sources of information co-determine activation of the word meaning (Harm \& Seidenberg, 2004; Grainger \& Ziegler, 2011). Harm and Seidenberg (2004), in particular, formalize a "cooperation" between a direct route that links orthography to meaning and an indirect route where word access is mediated by phonology, with the activation of semantic information being driven by the input coming from both the routes. This is in line with our results. Their model also assumes that the two routes democratically cooperate to determine word meaning, with one being more active when the other is less involved in the meaning computation. However, our findings suggest a slightly different 
Table 3 Results of the mediation analysis with 1,000 simulations

\begin{tabular}{llll}
\hline & Estimate & Lower 95 \% C.I. & Upper 95 \% CI \\
\hline A) PSC mediating the OSC effect (Fig. 2, panel 2a) & & 0.0098 \\
Total effect & -0.0299 & -0.0512 & -0.0068 \\
ACME (mediation effect) & -0.0232 & -0.0398 & 0.0189 \\
ADE (direct effect) & -0.0068 & -0.0326 & 2.5097 \\
Proportion mediated & 0.7735 & 0.1768 & 0.001 \\
B) OSC mediating the PSC effect (Fig. 2, panel 2b) & -0.0368 & -0.0565 & 0.0165 \\
Total effect & -0.0041 & -0.0196 & 0.0118 \\
ACME (mediation effect) & -0.0328 & -0.0601 & -0.0075 \\
ADE (direct effect) & 0.1107 & -0.3835 & 0.0589 \\
Proportion mediated & & 0.001 \\
\hline
\end{tabular}

The upper part of the table reports the results of a model in which PSC mediates the OSC effect. The lower part reports the results of a model in which PSC mediates the OSC effect. Quasi-Bayesian confidence intervals are reported. ACME, average causal mediation effect; ADE, average direct effect

picture: The results of the mediation analysis show that in order to access word meaning the relative contribution of orthography vis-a-vis phonology is in favor of the latter (i.e., most of the orthography-to-semantics effect could be actually explained in phonological terms). This suggests that readers tend to access semantics by means of the orthography-phonology-semantics pathway; the impact of the orthography-semantics route becomes evident only when the former is less efficient.

The application of our consistency measures also provides an important step forward with respect to traditional models. In fact, OSC and PSC quantify the relationship between form and semantics at a large-scale level, considering as source data a corpus of 2.8 billion tokens. In this perspective, it goes beyond the limitations of existing models on the role of phonology that mostly focus on small samples of short words (1 or 2 syllables) to support their claims.

One may ask why readers exploit associations between forms and meanings. Recently, it has been proposed that systematicity - defined as "a statistical relationship between the patterns of sound for a group of words and their usage" (Dingemanse, Blasi, Lupyan, Christiansen, \& Monaghan, 2015, p. 606) - may be a key feature in language. Statistical regularities between form (phonological or orthographic) and meaning assist category learning with the help of phonological cues and may support word identification. The orthographic similarity among stimuli with similar meaning will help the reader both to learn any novel item with similar (orthographic) properties and to recognize it. On the contrary, when an orthographic pattern is associated to several meanings, the extreme level of arbitrariness will make the processing more uncertain, because there are not cues on which to rely for semantics (for a similar explanation, see Marelli et al., 2015). The same reasoning applies also to the phonology-to-semantics relation, with the sound-meaning associations being benefit in terms of both word learning and recognition. In this perspective, the present results can be seen as a contribution to the more general evidence concerning the central role of systematicity in language, that is arguably the most important aspect for approaches to word comprehension that are based on learning mechanisms (Baayen, Milin, Đurđević, Hendrix \& Marelli, 2011).

To conclude, our results add to an already existing body of literature arguing in favor of phonological recoding in lexical access (Rastle \& Brysbaert, 2006) or semantic access (Van Orden, 1987). Furthermore, we provide a novel look at the old problem, proposing a new bottom-up measure that should be taken into account in future studies on reading.

Acknowledgments Author Contributions: SA, MM, and SS designed the study. SA and SS created the stimuli database; MM created the computational model; MM and SA ran the statistical analyses. SA, MM and SS drafted the paper. The authors thank Petar Milin and an anonymous reviewer for their insightful comments.

\section{References}

Arora, S., Ge, R., \& Moitra, A. (2012). Learning topic models-going beyond SVD. In Foundations of Computer Science (FOCS), 2012 I.E. 53rd Annual Symposium (pp. 1-10).

Bayen, R. H. (2008). Analyzing linguistic data: A practical introduction to statistics using $R$. New York, NY: Cambridge University Press.

Bayen, R. H., Davidson, D. J., \& Bates, D. M. (2008). Mixed-effects modeling with crossed random effects for subjects and items. Journal of Memory and Language, 59(4), 390-412. doi:10.1016/j. jml.2007.12.005

Baayen, R. H., Milin, P., Đurđević, D. F., Hendrix, P., \& Marelli, M. (2011). An amorphous model for morphological processing in visual comprehension based on naive discriminative learning. Psychological Review, 118(3), 438. doi:10.1037/a0023851

Baayen, R. H., Piepenbrock, R., \& Gulikers, L. (1995). The CELEX lexical database (CD Rom). University of Pennsylvania, Philadelphia, PA: Linguistic Data Consortium.

Balota, D. A., Yap, M. J., Hutchison, K. A., Cortese, M. J., Kessler, B., Loftis, B., ... \& Treiman, R. (2007). The English Lexicon Project. Behavior Research Methods, 39, 445-459. 
Braun, M., Hutzler, F., Münte, T. F., Rotte, M., Dambacher, M., Richlan, F., \& Jacobs, A. M. (2015). The neural bases of the pseudohomophone effect: Phonological constraints on lexicosemantic access in reading. Neuroscience, 295, 151-163. doi:10.1016/j.neuroscience.2015.03.035

Brysbaert, M., Praet, C., \& d'Ydewalle, G. (1990). Phonological recoding in reading isolated words: A secondary and optional channel. University of Leuven Technical Report, 110, 1-36.

Bullinaria, J. A., \& Levy, J. P. (2007). Extracting semantic representations from word co-occurrence statistics: A computational study. Behavior Research Methods, 39, 510-526.

Church, K., \& Hanks, P. (1990). Word Association Norms, Mutual Information, and Lexicography. Computational Lingistics, 16, 22-29.

Coltheart, V., Avons, S. E., Masterson, J., \& Laxon, V. J. (1991). The role of assembled phonology in reading comprehension. Memory \& Cognition, 19, 387-400.

Dingemanse, M., Blasi, D. E., Lupyan, G., Christiansen, M. H., \& Monaghan, P. (2015). Arbitrariness, Iconicity, and Systemacity in Language. Trends in Cognitive Science, 19, 603-615. doi:10.1016/j. tics.2015.07.013

Dinu, G., The Pham, N., \& Baroni, M. (2013). DISSECT: DIStributional SEmantics Composition Toolkit. In Proceedings of ACL (System Demonstrations) (pp. 31-36). Sofia, Bulgaria.

Frost, R. (1998). Toward a strong phonological theory of visual word recognition: True issues and false trails. Psychological Bulletin, $123,71$.

Grainger, J., \& Ziegler, J. C. (2011). A dual-route approach to orthographic processing. Frontiers in Psychology, 2, 54. doi:10. 3389/fpsyg.2011.00054

Harm, M. W., \& Seidenberg, M. S. (2004). Computing the meanings of words in reading: Cooperative division of labor between visual and phonological processes. Psychological Review, 111, 662-720.

Jared, D., \& Seidenberg, M. S. (1991). Does word identification proceed from spelling to sound to meaning? Journal of Experimental Psychology: General, 120, 358-394.

Keuleers, E., Lacey, P., Rastle, K., \& Brysbaert, M. (2012). The British Lexicon Project: Lexical decision data for 28,730 monosyllabic and disyllabic English words. Behavior Research Methods, 44, 287304. doi:10.3758/s13428-011-0118-4

Kuznetsova, A., Brockhoff, P. B., \& Christensen, R. H. B. (2015). Package 'ImerTest'. $R$ package version, 2-0.

Landauer, T., \& Dumais, S. (1997). A solution to Plato's problem: The latent semantic analysis theory of acquisition, induction and representation of knowledge. Psychological Review, 104, 211-240.
Lesch, M. F., \& Pollastek, A. (1993). Automatic access of semantic information by phonological codes in visual word recognition. Journal of Experimental Psychology: Learning, Memory, and Cognition, 19, 285-294.

Lund, K., \& Burgess, C. (1996). Producing high-dimensional semantic spaces from lexical cooccurrence. Behavioural Research Methods, 28, 203-208.

Marelli, M., Amenta, S., \& Crepaldi, D. (2015). Semantic transparency in free stems: The effect of Orthography-Semantics Consistency in word recognition. Quarterly Journal of Experimental Psychology, 68, 1571-1583. doi:10.1080/17470218.2014.959709

Marelli, M., \& Baroni, M. (2015). Affixation in semantic space: Modeling morpheme meanings with compositional distributional semantics. Psychological Review, 122(3), 485. doi:10. 1037/a0039267

Perfetti, C. A., Bell, L. C., \& Delaney, S. M. (1988). Automatic (prelexical) phonetic activation in silent word reading: Evidence from backward masking. Journal of Memory and Language, 27, 59-70.

Rastle, K., \& Brysbaert, M. (2006). Masked phonological priming effects in English: Are they real? Do they matter?. Cognitive Psychology, 53(2), 97-145. doi:10.1016/j.cogpsych.2006.01.002

Seidenberg, M. (1985). The time course of phonological code activation in two writing systems. Cognition, 19, 1-30.

Seidenberg, M. S. (1995). Visual word recognition. In J. L. Miller \& P. D. Eimas (Eds.), Handbook of perception \& cognition: Vol. 11. Speech, language \& communication (pp. 137-179). San Diego: Academic Press.

Tingley, D., Yamamoto, T., Hirose, K., Keele, L., \& Imai, K. (2014). Mediation: R package for causal mediation analysis. Journal of Statistical Software, 59. doi:10.18637/jss.v059.i05

Turney, P. D., \& Pantel, P. (2010). From frequency to meaning: Vector space models of semantics. Journal of Artificial Intelligence Research, 37, 141-188. doi:10.1613/jair.2934

Van Heuven, W. J., Mandera, P., Keuleers, E., \& Brysbaert, M. (2014). SUBTLEX-UK: A new and improved word frequency database for British English. The Quarterly Journal of Experimental Psychology, 67, 1176-1190. doi:10.1080/17470218.2013.850521

Van Orden, G. C. (1987). A ROWS is a ROSE: Spelling, sound, and reading. Memory \& Cognition, 15, 181-198.

Van Orden, G. C., Johnston, J. C., \& Hale, B. L. (1988). Word identification in reading proceeds from spelling to sound to meaning. Journal of Experimental Psychology: Learning, Memory, and Cognition, 14, 371. 